\title{
Impact of Treatment with Renin-Angiotensin System Inhibitors on Clinical Outcomes in Hypertensive Patients Hospitalized with COVID-19
}

\author{
Martin Negreira-Caamaño ${ }^{1}$. Jesus Piqueras-Flores ${ }^{1}$. Jorge Martínez-DelRio ${ }^{1}$. \\ Patricia Nieto-Sandoval-Martin-DeLaSierraa ${ }^{2}$ Daniel Aguila-Gordo ${ }^{1}$. Cristina Mateo-Gomez ${ }^{1}$. Daniel Salas-Bravo ${ }^{1}$. \\ Marta Rodriguez-Martinez ${ }^{2} \cdot$ Martín Negreira-Caamaño $^{1}[$
}

Received: 19 July 2020 / Accepted: 5 September 2020 / Published online: 19 September 2020

(c) Italian Society of Hypertension 2020

\begin{abstract}
Introduction Concerns have been raised about the possible harmfulness of angiotensin-converter enzyme inhibitors (ACEi) and aldosterone receptor blockers (ARB) in patients with COVID-19. However, few data from a European population have been published, especially from hypertensive patients.

Aim To study the association between ACEi or ARB treatments and major adverse outcomes during hospitalisation in COVID-19 patients.

Methods We studied 545 consecutive hypertensive patientsadmitted to our institution due to COVID-19 with respiratory involvement. Weanalysed the incidence of combined event (death or mechanical ventilatorysupport) during hospitalisation, as well as the time to independent events.

Results 188 (34.5\%) patients presentedthe combined endpoint. $182(33.4 \%)$ patients died, and $21(3.9 \%)$ neededmechanical ventilatory support. Patients with previous treatment with ACEi orARB presented similar incidence of the combined endpoint during hospitalisation( $31.6 \%$ vs. $41.8 \% ; p=0.08)$, with a lower all-cause mortality rate $(30.4 \%$ vs. $41.2 \% ; p=0.03)$ compared with those without prior treatment. Use of ACEi or ARB was not independentlyassociated with lower incidence of the combined endpoint [Adjusted OR 0.675 (95\% CI 0.298-1.528; $\mathrm{p}=0.146)$ ], but it was associated with lower mortality [Adjusted OR 0.550 (95\% CI 0.304-0.930; $\mathrm{p}=0.047$ )].

Conclusions The use of ACEi or ARB was associated with lessincidence of all-cause death during hospitalisation among hypertensive patientsadmitted with COVID-19 respiratory infection.
\end{abstract}

Keywords Hypertension · COVID-19 · Renin-angiotensin system (RAS) · Antihypertensive therapy · Angiotensinconverting enzyme inhibitors (ACEIs) · Angiotensin receptor blockers (ARBs)

\section{Introduction}

Electronic supplementary material The online version of this article (https://doi.org/10.1007/s40292-020-00409-7) contains supplementary material, which is available to authorized users.

Martín Negreira-Caamaño

martin.negcam@gmail.com

1 Cardiology Department, University General Hospital of Ciudad Real, Avda. del Obispo Rafael Torija, s/n, 13005 Ciudad Real, Spain

2 Pharmacy Department, University General Hospital of Ciudad Real, Ciudad Real, Spain
In December of 2019, a new type of coronavirus (severe acute respiratory syndrome coronavirus 2 , SARS-CoV-2) was reported in Wuhan [1]. Responsible of the coronavirus disease 2019 (COVID-19), the virus promptly spread worldwide, causing more than 100,000 deaths in the first 6 months after the first reports. The disease was rapidly recognised as systemic [2]. Although the respiratory system is most frequently affected in COVID-19, a high incidence of cardiovascular events has also been noticed [3, 4].

The pathophysiology of COVID-19 is not yet well understood. However, the role of the renin-angiotensin system (RAS) has been proved to be relevant in infection 
mechanisms and disease severity, as was previously described with other coronavirus infections [5].

The angiotensin-converter enzyme (ACE) 2 is closely implicated in the virus infection $[6,7]$. This molecule is widely expressed all over the human body (i.e., alveolar cells, kidney, gastrointestinal tract, heart, vascular endothelium). Once the virus has penetrated the cellular membrane, the ACE- 2 receptor reduces its expression on the cellular surface. By the inhibition of ACE 1, as with angiotensin receptor blockers (ARB) or ACE inhibitors (ACEi), an overexpression of ACE2 that facilitates the infection could be promoted [8]. Some reports have correlated the use of RAS inhibitors with increased concentrations of ACE-2 [9], raising concerns about individual susceptibility to SARS-CoV-2 infection and propagation in patients under these therapies. On the other hand, a recent study suggested that this effect would be associated with the use of mineralocorticoid receptor antagonist [10], but not with ACEi or ARB use.

Arterial hypertension (AH) has been described as an important risk factor for SARS-CoV-2 infection, as well as a predictor of worse outcomes [11]. ACEi and ARB are firstline drugs recommended in $\mathrm{AH}$ management, but the effect of these therapies in terms of prognosis remains unclear in patients with COVID-19, with some studies reporting neutral (or even beneficial) effects [11-13], while others have noted adverse outcomes $[14,15]$.

Remarkably, compared to other southern European countries (e.g., Italy), Spain remains one of the countries with the highest infection and mortality rates due to COVID-19, with more than 240,000 confirmed infections and 28,000 deaths at the time we wrote this document $[16,17]$.

In this document, we aim to analyse the association between the use of RAS inhibitors and in-hospital outcomes in a Spanish cohort of hypertensive patients.

\section{Methods}

\subsection{Patients and Study Design}

This is a single-centre observational cohort study based on the consecutive enrolment of patients with SARS-CoV-2 infection admitted to our institution between March 3rd and April 30th of 2020. Exclusion criteria were: age under 18 years old, no history of $\mathrm{AH}$, negative protein-chain-reaction test results, and absence of respiratory involvement. The study flowchart is showed in Fig. 1.

We analysed the incidence of severe adverse events during hospitalisation and evaluated possible differences between patients with prior ACEi or ARB prescription and the rest of hypertensive patients.

\subsection{Data Collection}

We recorded demographic, clinical, and analytical variables, as well as the development of relevant clinical events during the follow-up.

Past medical history and clinical information were obtained from electronic medical records. Previous medical prescriptions and in-hospital medical therapies were confirmed using the current specific prescription software in our health area. All X-ray studies were assessed by an independent investigator and classified as unilateral, bilateral, or non-pulmonary infiltrates. Laboratory data were obtained from specific local software of our institution.

\subsection{Definitions}

Hypertensive patients were defined as those with previously established diagnoses of hypertension in their medical records or confirmed use of antihypertensive drugs for at least 1 month before hospital admission. We considered as antihypertensive drugs ACEi, ARB, loop diuretics, thiazides, beta blockers, aldosterone antagonists, calcium channel antagonists, and $\alpha$-blockers for cardiovascular indications.

Admission criteria were determined by an emergency department physician following the recommendations of the Health Ministry of the Government of Spain, which included the presence of respiratory failure (defined as arterial oxygen saturation $<90 \%$ or arterial oxygen tension $<90 \mathrm{mmHg}$ ), tachypnoea (defined as $>30$ breaths per minute), pulmonary infiltrates on X-ray or tomography studies, or coexistence of other systems' impairment.

The primary endpoint was defined as the incidence of the combined event [all-cause death or need for mechanical ventilation support (MVS)] during hospitalisation. Secondary endpoints included the independent incidence of death or VMS and the time to each event.

The follow-up period was measured in days from hospital admission to the date of the clinical event or to hospital discharge if no events were registered.

\subsection{In-Hospital Treatment and Discharge Criteria}

Treatments during hospitalisation were prescribed by the physician in charge of the patient following a local protocol approved by the institution's ethical committee. 
Fig. 1 Study flowchart. $A C E i$ angiotensin converter enzyme inhibitors, $A R B$ angiotensin-II receptor blockers, $P C R$ protein chain reaction, $S A R S-C O V 2$ severe acute respiratory syndrome coronavirus 2

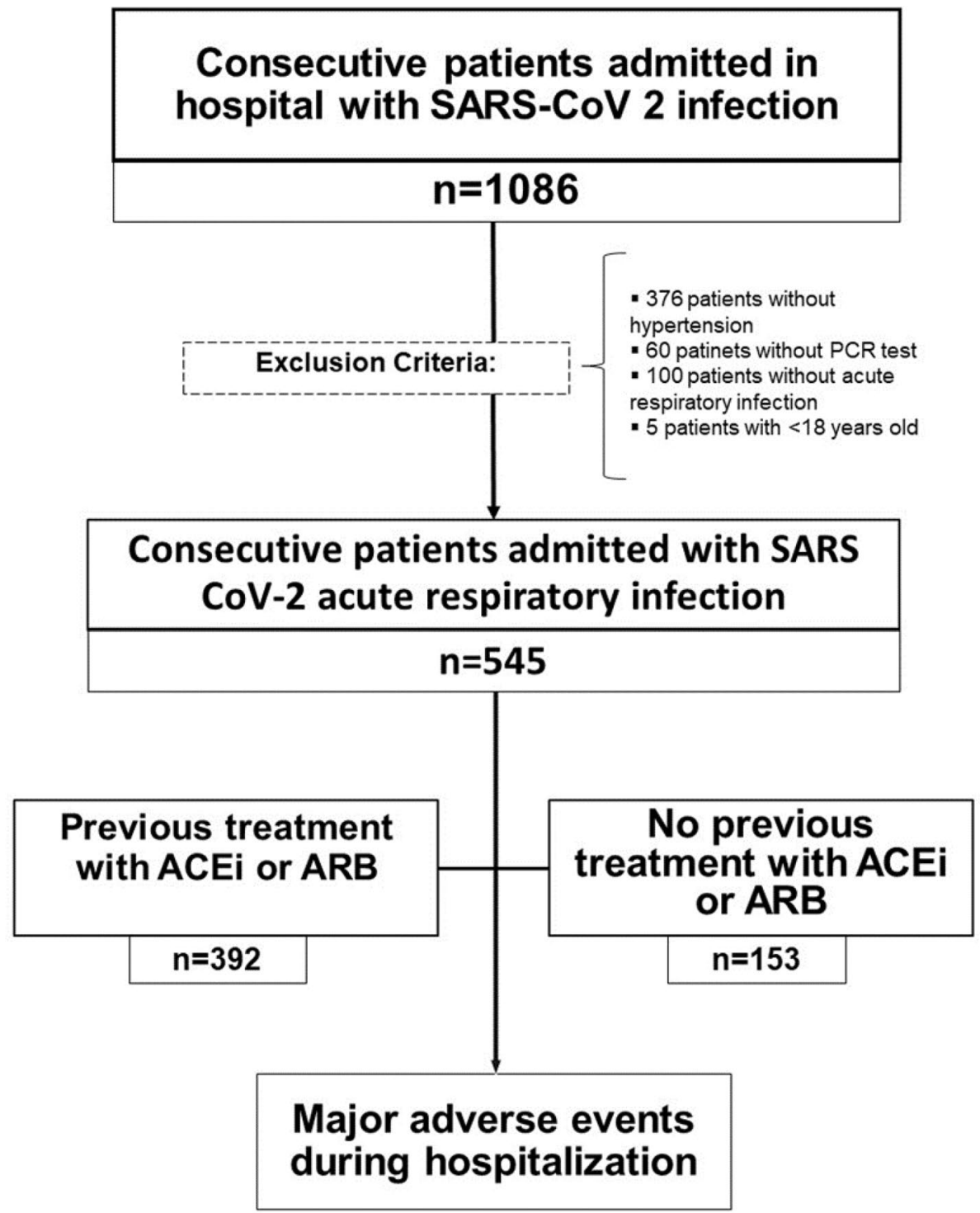

\subsection{Ethical Statement}

The study protocol was performed according to the Declaration of Helsinki and was approved by an ethical committee. Data were recorded using a dissociate-identity model to preserve the anonymity of the enrolled patients.

\subsection{Statistical Analysis}

Gaussian or non-Gaussian distribution was evaluated by the Kolmogorov-Smirnov test. Quantitative variables that follow a normal distribution are expressed as mean \pm standard deviation, and those with non-Gaussian distribution as median (interquartile range). Qualitative variables are expressed as percentages. For comparisons between quantitative variables, Student's t test or the Wilcoxon test was used. Qualitative variables were compared using the $\chi^{2}$ test or McNemar's test. A two-tailed probability value of $\leq 0.05$ was considered significant, and all confidence intervals were computed at the $95 \%$ confidence interval (CI). Time to event was analysed using a Kaplan-Meier model, and groups were compared using the log-rank test. A multivariable model was performed including all the unequally distributed $(\mathrm{p}<0.05)$ variables in the univariate analysis. A logistic regression model was implemented for the binary endpoints and a Cox proportional hazards model for the survival endpoints. The statistical analysis was performed using the SPSS version 25.0 (IBM, New York). 


\section{Results}

\subsection{Participants}

From 3 March to 30 April 2020, 1086 patients were admitted to our institution with diagnosis of SARS-CoV-2 infection. Five hundred forty-one patients met the exclusion criteria and 545 patients were included in subsequent analysis (Fig. 1). Among them, 392 were classified as the ACEi/ARB group, and 153 were categorised as the nonACEi/ARB group.

The mean age was $76.5 \pm 12.3$ years, and nearly $50 \%$ of patients were male. The most prevalent cardiovascular risk factor was diabetes $(30.1 \%)$, although a high prevalence of other comorbid conditions, such as chronic lung disease $(22.6 \%)$ or chronic kidney disease $(18.0 \%)$, was found. Baseline characteristics of the study cohort are presented in Table 1.

\subsection{Previous Antihypertensive Treatment}

The most frequently used antihypertensive drug was ARB (used by the $43.5 \%$ of the patients), followed by thiazides (30.5\%) and beta blockers (29.2\%). Among those patients who were treated with ACEi/ARBs, fewer were concurrently treated with loop diuretics, beta blockers, and aldosterone antagonists, and more were treated with thiazides (Table 2).

\subsection{Clinical and Biochemical Characteristics at Admission and During Hospitalisation}

Most patients were admitted within the first 7 days after symptoms' onset (63.1\%) and had bilateral lung consolidation in the first radiological study (72.7\%). Respiratory clinical repercussion was frequent, and the median CURB65 score was $1.9 \pm 1.2$, similar between groups. Nevertheless, no differences were observed in clinical manifestations at hospital admission between the ACEi/ARB and nonACEi/ARB groups. Clinical and biochemical data regarding in-hospital admission are shown in the Supplementary Material.

Most patients presented high levels of analytical inflammatory markers at admission: $87 \%$ had elevated C-reactive protein, $79.3 \%$ had abnormal D-dimer values, and $90.4 \%$ had high fibrinogen levels. Cytolysis enzymes were less frequently elevated. Notably, high-sensitivity troponin was requested for a small percentage of patients (4.8\%). We did not observe significant differences in biochemical parameters when comparing the ACEi/ARB group to the non-ACEi/ ARB group (Supplementary Material).

\subsection{In-Hospital Therapies}

During hospitalisation, the most frequently prescribed antihypertensive treatment was calcium channel blockers (54.4\%), followed by ACEi or ARB (30.8\%). Remarkably, $16.1 \%$ of patients did not received any antihypertensive therapy during hospitalisation, although only $5 \%$ of
Table 1 Baseline characteristics of the study cohort

\begin{tabular}{lllll}
\hline & Overall $(\mathrm{n}=545)$ & ACEi/ ARB $(\mathrm{n}=392)$ & $\begin{array}{l}\text { No ACEi/ARB } \\
(\mathrm{n}=153)\end{array}$ & $\mathrm{p}$ \\
\hline Male sex (\%) & $283(51.9)$ & $206(52.6)$ & $77(50.3)$ & 0.640 \\
Age (years) & $76.5 \pm 12.3$ & $75.9 \pm 12.1$ & $78.0 \pm 12.9$ & 0.076 \\
Diabetes mellitus (\%) & $165(30.1)$ & $125(31.9)$ & $40(26.1)$ & 0.366 \\
History of smoking (\%) & $99(18.2)$ & $70(17.9)$ & $29(19.0)$ & 0.675 \\
Obesity (\%) & $108(19.8)$ & $81(20.8)$ & $27(17.6)$ & 0.427 \\
Lung disease (\%) & & & $17(11.1)$ & 0.236 \\
COPD & $48(8.8)$ & $31(7.9)$ & $5(3.3)$ & 0.569 \\
Asthma & $22(4.0)$ & $17(4.3)$ & $14(9.1)$ & 0.771 \\
$\quad$ OSAS & $53(9.7)$ & $39(9.9)$ & $25(16.3)$ & 0.128 \\
Heart failure (\%) & $70(12.8)$ & $45(11.5)$ & $7(4.6)$ & 0.332 \\
Reduced LVEF (\%) & $18(3.3)$ & $11(2.8)$ & $16(10.5)$ & 0.334 \\
Ischemic heart disease (\%) & $69(12.7)$ & $53(13.5)$ & $33(21.6)$ & 0.016 \\
Atrial fibrillation (\%) & $85(15.6)$ & $52(13.3)$ & $33(21.7)$ & 0.162 \\
Chronic kidney disease (\%) & $98(18.0)$ & $65(16.6)$ & $6(3.9)$ & 0.592 \\
Active cancer (\%) & $27(5.0)$ & $21(5.4)$ & $4.8 \pm 2.1$ & 0.082 \\
Charlson Comorbidity Index & $4.6 \pm 2.1$ & $4.5 \pm 2.2$ & & \\
\hline
\end{tabular}

$A C E i$ angiotensin converter enzyme inhibitors, $A R B$ angiotensin receptor blockers, $C O P D$ chronic obstructive pulmonary disease, $L V E F$ left ventricle ejection fraction, $O S A S$ obstructive sleep apnea syndrome 
Table 2 Previous and in-hospital antihypertensive therapies

\begin{tabular}{|c|c|c|c|c|}
\hline & Overall $(\mathrm{n}=545)$ & ACEi/ARB $(n=392)$ & $\begin{array}{l}\text { No ACEI/ARB } \\
(\mathrm{n}=153)\end{array}$ & $\mathrm{p}$ \\
\hline \multicolumn{5}{|l|}{ Previous admission Ah therapies } \\
\hline Loop diuretic $(\%)$ & $130(23.9)$ & $86(21.9)$ & $44(28.8)$ & 0.093 \\
\hline Thiazide diuretic (\%) & $166(30.5)$ & $151(38.5)$ & $15(9.8)$ & $<0.001$ \\
\hline Betablocker (\%) & $159(29.2)$ & $100(25.5)$ & $59(38.6)$ & 0.003 \\
\hline Calcium channel antagonist (\%) & $141(25.9)$ & $97(24.7)$ & $44(28.8)$ & 0.336 \\
\hline Mineralocorticoid receptor antagonist (\%) & $34(6.2)$ & $18(4.6)$ & $16(10.5)$ & 0.011 \\
\hline Doxazosin $(\%)$ & $45(8.3)$ & $29(7.4)$ & $16(10.5)$ & 0.244 \\
\hline \multicolumn{5}{|l|}{ In Hospital AH therapies } \\
\hline Any & $457(83.9)$ & $392(100)$ & $119(77.8)$ & $<0.001$ \\
\hline AH without treatment & $34(6.2)$ & $0(0.0)$ & $34(22.2)$ & $<0.001$ \\
\hline ACEi or ARB & $168(30.8)$ & $150(38.3)$ & $\begin{array}{l}18(11.8) \\
5(3.6)\end{array}$ & $<0.001$ \\
\hline Mineralocorticoid antagonist (\%) & $22(4.0)$ & $14(3.6)$ & $8(5.2)$ & 0.380 \\
\hline Calcium channel antagonist (\%) & $296(54.4)$ & $236(60.4)$ & $60(39.2)$ & $<0.001$ \\
\hline Diuretics (\%) & $154(28.3)$ & $102(26.0)$ & $52(34.0)$ & 0.063 \\
\hline Beta blockers (\%) & $142(26.1)$ & $95(24.3)$ & $47(30.7)$ & 0.125 \\
\hline Doxazosin $(\%)$ & $36(6.6)$ & $25(4.6)$ & $11(2.0)$ & 0.737 \\
\hline
\end{tabular}

ACEi angiotensin converter enzyme inhibitors, $A R B$ angiotensin receptor blockers, $A H$ arterial hypertension; $n . a$ not applicable

patients were admitted with hypotension. This scenario was more frequently found in the non-ACEi/ARB group $(12.8 \%$ vs. $21.8 \%$; $\mathrm{p}<0.001)$. By contrast, the ACEi/ARB group showed a higher prescription rate of RAS inhibitors $(38.3 \%$ vs. $11.8 \% ; \mathrm{p}<0.001)$ and calcium channel blockers $(60.4 \%$ vs. $39.2 \%$; $<<0.001)$ during hospitalisation.

All patients received COVID-19-specific treatment for the disease, hydroxychloroquine being the most frequently used. Pharmacological treatment prescribed during hospitalisation is shown in the Supplementary Material. No differences were observed in COVID-19-specific therapy prescription, but in-hospital treatment with lopinavir-ritonavir was more frequent in the ACEi/ARB group (37.2\% vs. $26.1 \% ; \mathrm{p}=0.014)$.

\subsection{Adverse Events During Hospitalisation}

After a median time of hospitalisation of 7 days (5-11 days), $188(34.5 \%)$ patients presented the combined endpoint. The ACEi/ARB group showed a trend for a lower incidence of the composite endpoint ( $31.6 \%$ vs. $41.8 \%$; $p=0.080$ ).

After multivariable analysis, previous treatment with ACEi or ARB was not independently associated with the primary endpoint [Adjusted OR 0.675 (95\% CI 0.298-1.528; $\mathrm{p}=0.146)]$.

The Kaplan-Meier analysis did not show differences in the cumulative incidence of the combined event between ACEi/ARB and non-ACEi/ARB groups $(p=0.08)$ (Fig. 2).
One hundred eighty-two (33.4\%) patients died during hospitalisation, and 21 (3.9\%) needed MVS. Patients with previous ACEi/ARB treatment presented a lower all-cause death rate [OR: 0.623 (95\% CI 0.423-0.917; $\mathrm{p}=0.017)$ ], but no differences were observed in the need of MVS [OR: 3.846 (95\% CI 0.885-16.714; $\mathrm{p}=0.072$ )]. Similarly, the survival analysis showed that time to all-cause death was significantly shorter in patients without previous ACEi/ ARB treatment $(19.1 \pm 1.6$ vs. $22.3 \pm 1.4$ days; $p=0.030)$, but no differences were observed regarding time to MVS $(p=0.059)$. The event rate during hospitalisation and the survival analysis results are presented in Table 3 and Fig. 2.

After multivariable analysis, the previous treatment with ACEi or ARB remained an independent protective factor of in-hospital death [Adjusted OR 0.550 (95\% CI 0.304-0.930; $\mathrm{p}=0.047)$, although differences were not observed in the Cox regression model $(\mathrm{p}=0.122)$. All the variables included in the logistic regression models are shown in the Supplementary Material.

\section{Discussion}

The main finding of this study is that antihypertensive therapy with ACEI or ARB was associated with a beneficial effect regarding in-hospital major adverse outcomes in hypertensive patients admitted due to COVID-19. This finding was derived from the lower all-cause mortality rate among the patients treated with ACEi or ARB. 


\section{Kaplan-Meier analysis of major adverse events}

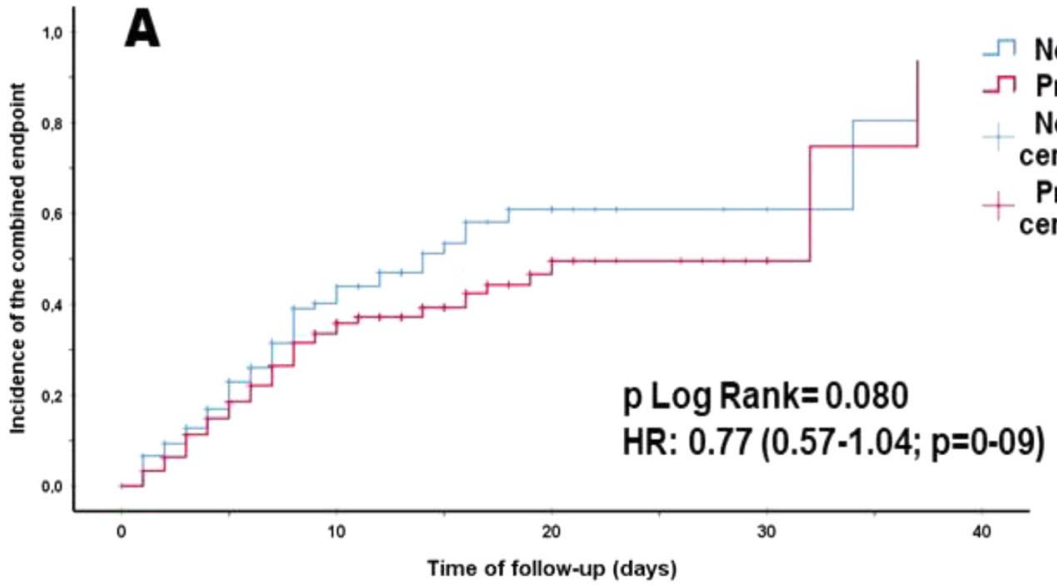

No previous ACEi/ ARB

Previous ACEi/ ARB

No previous ACEi/ ARB -

censored

Previous ACEi/ ARB -

censored

p Log Rank $=0.080$

HR: 0.77 (0.57-1.04; $p=0-09)$
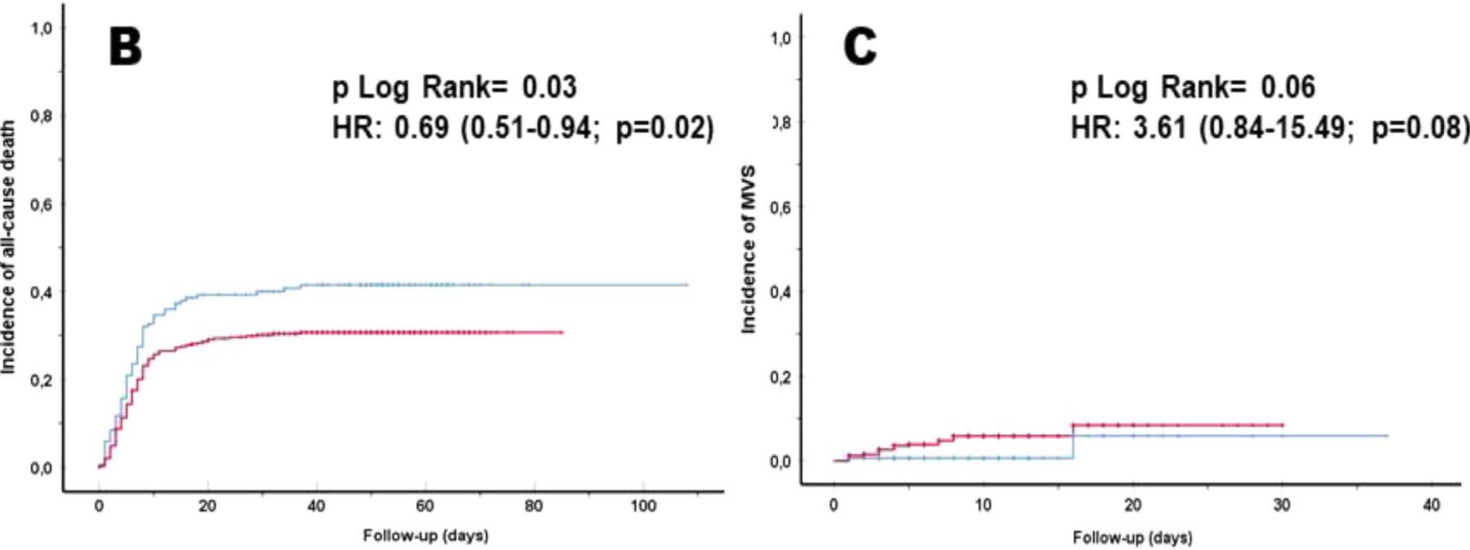

Fig. 2 Kaplan Meier curves of major adverse events during hospitalization: primary combined endpoint (a), all-cause mortality (b) and need of mechanical ventilatory support (c). ACE $i$ angiotensin converter enzyme inhibitors, $A R B$ angiotensin-II receptor blockers, $H R$ hazard ratio

Table 3 Primary and secondary outcomes during hospitalization

\begin{tabular}{|c|c|c|c|c|c|c|}
\hline & Overall $(n=545)$ & ACEi/ARB $(n=392)$ & $\begin{array}{l}\text { No ACEI/ARB } \\
(\mathrm{n}=153)\end{array}$ & $\mathrm{p}$ & OR $(95 \% \mathrm{CI})$ & $\mathrm{p}^{\&}$ \\
\hline Primary endpoint (\%) & $188(34.5)$ & $124(31.6)$ & $64(41.8)$ & 0.024 & $0.643(0.438-0.946)$ & 0.025 \\
\hline Death $(\%)$ & $182(33.4)$ & $119(30.4)$ & $63(41.2)$ & 0.016 & $0.623(0.423-0.917)$ & 0.017 \\
\hline \multirow[t]{2}{*}{ Need for MVS (\%) } & $21(3.9)$ & $19(4.8)$ & $2(1.3)$ & 0.054 & $3.846(0.880-16.714)$ & 0.072 \\
\hline & Overall $(n=545)$ & ACEi/ARB $(n=392)$ & $\begin{array}{l}\text { No ACEI/ARB } \\
(\mathrm{n}=153)\end{array}$ & $\mathrm{p}^{+}$ & HR $(95 \%$ CI $)$ & $\mathrm{p}^{\#}$ \\
\hline Time to primary endpoint (days) & $20.9 \pm 1.0$ & $21.6 \pm 1.4$ & $18.8 \pm 1.6$ & 0.080 & $0.770(0.568-1.042)$ & 0.089 \\
\hline Time to death (days) & $21.6 \pm 1.1$ & $22.3 \pm 1.4$ & $19.1 \pm 1.6$ & 0.030 & $0.689(0.508-0.935)$ & 0.017 \\
\hline Time to need for MVS (days) & $34.8 \pm 0.6$ & $28.1 \pm 0.5$ & $27.2 \pm 1.4$ & 0.059 & $3.608(0.840-15.493)$ & 0.085 \\
\hline
\end{tabular}

$A C E i$ angiotensin converter enzyme inhibitors, $A R B$ angiotensin-II receptor blockers, $M V S$ mechanical ventilatory support

${ }^{+} \mathrm{p}$ value of $\log$-rank test; ${ }^{\mathrm{p}} \mathrm{p}$ value of odds ratio; ${ }^{*} \mathrm{p}$ value of hazard ratio

Our results support recent findings in an Asian population that found a beneficial effect of treatment with ACEi or ARB prior to COVID-19 admission among patients with AH [11]. Regarding the need for mechanical ventilatory support, we did not find other studies in hypertensive patients to which to compare our results.

This study highlights the differences in COVID-19 severity and $\mathrm{AH}$ prevalence and therapy between the Asiatic and 
European populations. We found a markedly higher prevalence of AH among COVID-19 patients than previous studies from China, which found prevalence between 15 and $31 \%$ in admitted patients with COVID-19 [1, 11, 18, 19]. This could represent the higher AH prevalence among the European population when compared to the Chinese but also could be a result of the older population of our study. Referring to $\mathrm{AH}$ therapy, the most frequent drugs prescribed in our cohort were those endorsed by the current international recommendations, suggesting an optimally treated population [14]. The most frequent AH therapy substantially varies from Chinese to European reports, with our results being concordant with usual European AH therapies. In Europe, $\mathrm{ACEi}$ and $\mathrm{ARB}$ are the most frequent antihypertensive therapy, while in Asia, the calcium channel option seems to be more attractive $[20,21]$.

One of the most impactful results is the substantial increase in mortality when we compared our data with those of other countries, which had mortality rates around $10 \%$ among AH patients. This could be highly influenced by the age of our population, almost 10 years older than that of other studies [11, 12, 22], as well as due to a higher prevalence of comorbidities associated with worse prognosis, such as asthma or chronic kidney disease. This hypothesis is supported by observational studies in China, with older cohort, where AH patients had up to $44 \%$ mortality [21].

Notably, we observed a high percentage of patients in whom, despite a lack of hypotension at admission, antihypertensive therapy was retired. This scenario could have been influenced by messages of alarm on social media and among the scientific community about ACEi and ARB therapy in patients with COVID-19 [23]. On the other hand, a small proportion of patients started an ACEi or ARB.

To our knowledge, and regarding the geographical relevance, our study includes the largest cohort of hypertensive patients with COVID-19 in Europe. Spain is one of the most affected countries in terms of mortality due to COVID19. With more than 45,000 deaths and more than 400,000 infected citizens, the country is the second in Europe, close to the United Kingdom [16]. Remarkably, our institution is the reference centre of the area with the highest mortality in Spain, with 200.9 deaths per 100,000 habitants [24].

To summarise, ACEi and ARB were not associated with worse outcomes in a large cohort of Mediterranean hypertensive patients admitted with COVID-19 respiratory infection, and no differences were observed between both treatments.

\subsection{Study Limitations}

This is an observational study with all the limitations that implies. We noted that patients could have had suboptimal treatment adherence prior to admission, as we only considered drug prescriptions. Moreover, the absence of patients with COVID-19 but without respiratory infection could have influenced the results and must be taken under consideration. Notably, we have suffered an abnormally high demand for critical care measures during the COVID-19 pandemic, which could have influenced some clinical decisions. In that way, the real rate of patients that could have received mechanical ventilatory support might have been underestimated, especially in the elderly population.

\section{Conclusion}

Among hypertensive patients with COVID-19, those treated with ACEi or ARB did not present greater risk of all-cause death or need for mechanical ventilatory support during hospitalisation. Indeed, these therapies could even be associated with a better prognosis.

Acknowledgements The authors wished to appreciate the daily labour of the workers in our institution. We are living in difficult times and the personal involvement of healthcare professionals is allowing the system to take a little breath beyond recent misfortunes.

\section{Compliance with Ethical Standards}

Conflict of interest The authors have no conflict of interest regarding the present investigation work.

Ethics approval The study was approved by an Ethics Committee that declined the need of informed consent due to the observational nature of the study and the pandemic situation, recognising an increased risk of infection due to unnecessary human contact.

Consent to participate Not applicable.

Consent for publication Not applicable.

Code availability The datasets generated during and/or analysed during the current study are available from the corresponding author on reasonable request.

\section{References}

1. Huang C, Wang Y, Li X, Ren L, Zhao J, Hu Y, et al. Clinical features of patients infected with 2019 novel coronavirus in Wuhan, China. Lancet. 2020;15(10223):497-506.

2. Lai CC, Ko WC, Lee PI, Jean SS, Hsueh PR (2020) Extra-respiratory manifestations of COVID-19. Int J Antimicrob Agents

3. Xiong T-Y, Redwood S, Prendergast B, Chen M. Coronaviruses and the cardiovascular system: acute and long-term implications. Eur Heart J. 2020 Mar;18(19):1798-800. 41(.

4. Jaffe AS, Cleland JGF, Katus HA. Myocardial injury in severe COVID-19 infection. Eur Heart J. 2020;41(22):2080-2.

5. Jaffe AS, Cleland JGF, Katus HA. Myocardial injury in severe COVID-19 infection. Eur Heart J. 2020;41(22):2080-2. 
6. Song W, Gui M, Wang X, Xiang Y. Cryo-EM structure of the SARS coronavirus spike glycoprotein in complex with its host cell receptor ACE2. PLOS Pathogens. 2018;14(8):e1007236.

7. Hoffmann M, Kleine-Weber H, Schroeder S, Krüger N, Herrler $\mathrm{T}$, Erichsen S et al (2020) SARS-CoV-2 cell entry depends on ACE2 and TMPRSS2 and is blocked by a clinically proven protease inhibitor. Cell 181(2):271-280.e8

8. Kuster GM, Pfister O, Burkard T, Zhou Q, Twerenbold R, Haaf $\mathrm{P}$, et al. SARS-CoV2: should inhibitors of the renin-angiotensin system be withdrawn in patients with COVID-19? Pathophysiology. Eur Heart J. 2020;41:1801-3.

9. Ferrario CM, Jessup J, Chappell MC, Averill DB, Brosnihan KB, Tallant EA, et al. Effect of angiotensin-converting enzyme inhibition and angiotensin II receptor blockers on cardiac angiotensinconverting enzyme 2. Circulation. 2005;111(20):2605-10.

10. Sama IE, Ravera A, Santema BT, et al. Circulating plasma concentrations of angiotensin-converting enzyme 2 in men and women with heart failure and effects of renin-angiotensin-aldosterone inhibitors. Eur Heart J. 2020;41(19):1810-7.

11. Gao C, Cai Y, Zhang K, Zhou L, Zhang Y, Zhang X, et al. Association of hypertension and antihypertensive treatment with COVID-19 mortality: a retrospective observational study. Eur Heart J. 2020;41(22):2058-66.

12. Zhang P, Zhu L, Cai J, Lei F, Qin J-J, Xie J, et al. Association of inpatient use of Angiotensin-converting enzyme inhibitors and Angiotensin II receptor blockers with mortality among patients with hypertension hospitalized with COVID-19. Circ Res. 2020;5(12):1671-81.

13. Khera R, Clark C, Lu Y, Guo Y, Ren S, Truax B et al (2020) Association of angiotensin-converting enzyme inhibitors and angiotensin receptor blockers with the risk of Hospitalization and Death in Hypertensive Patients with Coronavirus Disease-19. MedRxiv: the preprint server for health sciences Cold Spring Harbor Laboratory Press (2020.05.17.20104943)

14. Williams B, Mancia G, Spiering W, Agabiti Rosei E, Azizi M, Burnier M, et al. 2018 ESC/ESH Guidelines for the management of arterial hypertension: the Task Force for the management of arterial hypertension of the European Society of Cardiology
(ESC) and the European Society of Hypertension (ESH). Eur Heart J. 2018;25(33):3021-104.

15. Sommerstein R, Kochen MM, Messerli FH, Gräni C. Coronavirus Disease 2019 (COVID-19): do Angiotensin-converting enzyme inhibitors/angiotensin receptor blockers have a biphasic effect? J Am Heart Assoc. 2020;9(7):e016509.

16. Dong E, Du H, Gardner L (2020) An interactive web-based dashboard to track COVID-19 in real time. Lancet Infect Dis. http:// coronavirus.jhu.edu/map/html.

17. Iaccarino G, Borghi $C$, Cicero AFG, Ferri C, Minuz $P$, Muiesan ML, et al (2020) Renin-angiotensin system inhibition in cardiovascular patients at the time of COVID19: much Ado for Nothing? A statement of activity from the Directors of the Board and the Scientific Directors of the Italian Society of Hypertension. High Blood Press Cardiovasc Prev 27(2):105-8.

18. Guan W, Ni Z, Hu Y, Liang W, Ou C, He J, et al. Clinical characteristics of Coronavirus Disease 2019 in China. N Engl J Med. 2020;30(18):1708-20.

19. Li J, Wang X, Chen J, Zhang H, Deng A (2020) Association of Renin-Angiotensin system inhibitors with severity or risk of death in patients with hypertension Hospitalized for Coronavirus Disease 2019 (COVID-19) infection in Wuhan, China. JAMA Cardiol:23

20. Chow CK, Teo KK, Rangarajan S, Islam S, Gupta R, Avezum A, et al. Prevalence, awareness, treatment, and control of hypertension in rural and urban communities in high-, middle-, and lowincome Countries. JAMA. 2013;4(9):959-68.

21. Zengwu W, Zuo C, Linfeng Z, Xin W, Guang H, Zugui Z, et al. Status of hypertension in China. Circulation. 2018;29(22):2344-56.

22. Iaccarino G, Grassi G, Borghi C, et al. Age and multimorbidity predict death among COVID-19 patients: results of the SARSRAS study of the Italian Society of Hypertension. Hypertension. 2020;76(2):366-72.

23. Fang L, Karakiulakis G, Roth M. Are patients with hypertension and diabetes mellitus at increased risk for COVID-19 infection? Lancet Respir. 2020;8:e21.

24. Situación de (2020) COVID-19 en España. Ministerio de Sanidad. Consulted 2020 Jun 12. https://covid19.isciii.es. 